\title{
Comparative Study of Multiband Antennas With Fractal Geometry
}

\author{
Rekha G Nair \\ Assistant Professor, Department of Electronics \& Communication Engineering, NSS College of Engineering,
}

Palakkad, Kerala, India

\begin{abstract}
Main objective of wireless communication system is the design of wideband, multiband low profile small antenna. A fractal antenna effectively utilizes the available space within a sphere of radius ' $r$ ' which improves the bandwidth of the antenna enclosed in the sphere [2]. The term fractal refers to miniaturized structure which stands for irregular or broken pieces. Several natural phenomenons like trees, mountains etc. shows fractal nature. The fractal concepts are used in antenna theory and design and there are many studies and implementation of fractal antenna elements and arrays. This paper brings a comparison of different shrinking methods on antenna size based on fractal geometry while maintaining performance parameters. This paper presents a survey on fractal antennas designed with different geometric structures, its iteration process, radiation parameters etc. Which are used for wireless applications.
\end{abstract}

Keywords: Fractal Geometry, Sierpinski gasket, Koch Fractal, Hilbert Curve.

\section{INTRODUCTION}

The term FRACTAL, coined by Benoit B. Mandelbrot iteration is carried out ' $n$ ' number of times which refers to (French Mathematician) in 1970's from a Latin word the order of Sierpinski-gasket fractal. As in Figure1, the "fractus", means "fractured" or "broken" [1]. Fractal black triangle areas represent a metallic conductor whereas concepts have been applied to many branches of science the regions where metal has been removed are represented and engineering including antenna theory and design. by the white triangle areas. The formula for total length of Fractal antenna finds this wide spread application because Sierpinski gasket is $1=h(3 / 2)^{\mathrm{n}}$, where $\mathrm{h}$ is the curve height of its multiband property and small physical size, as fractals contains scaled copies of itself it can operate at different wave lengths hence it exhibits the multi band property keeping the radiation parameters similar at different operating wavelengths. The self similarity exhibited by fractal, effectively utilizes the available space. Fractals can be classified into deterministic and random. Deterministic contain scaled down and rotated versions of themselves, [2]. Random fractals have some elements of randomness. Deterministic fractals are again classified into linear and nonlinear. Fractal geometries can be generated by iterative process which results in self similar properties with Minkowski Island, Koch fractal loop, Sierpinski gaskets etc. By effectively utilizing the space filling properties fractal antennas overcome some of the limitations of electrically small antennas that they can resonate and show much higher input resistance than antennas with classic geometry.

\section{LITERATURE SURVEY}

Adopting Fractals is one way in which antenna size can be reduced. The researches in the field of fractal antennas resulted in tremendous growth in antenna design technology and hence the field of wireless communication. A survey on different previously proposed antennas based on fractal geometries is carried out here.

\subsection{Sierpinski gasket}

The first fractal geometry to be described is the Sierpinski gasket [3]. The procedure for constructing this geometry of fractal begins with an equilateral triangle and then removing the similar triangle from the former triangle, the

and " $n$ " is the number of iterations.
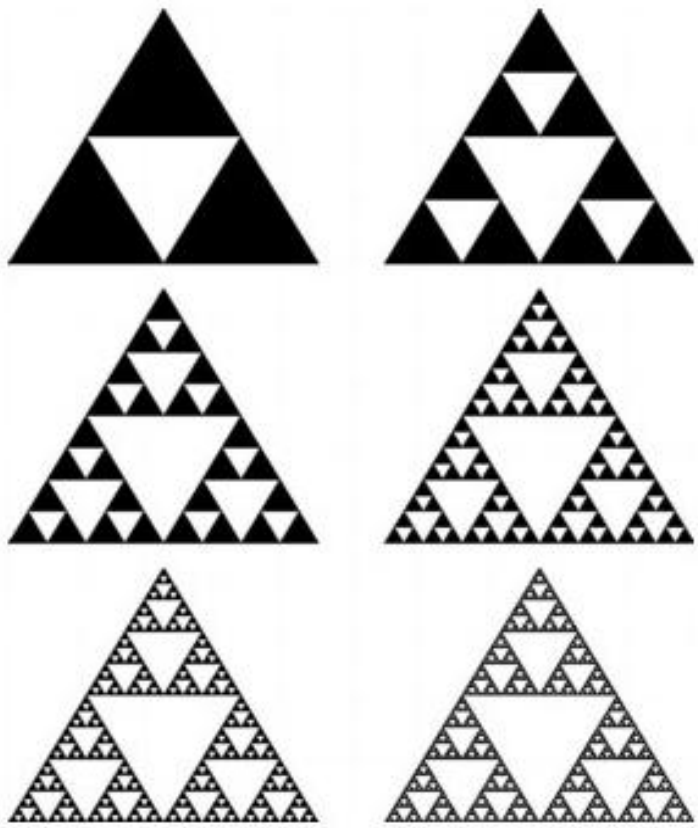

Figure .1

\subsection{Koch Fractal}

Here the iteration starts with a Euclidean Triangle and is portioned into three equal parts and the segment at middle is replaced with two others of same length to form the generator. This is reused in higher iteration, keeping scaled versions of the imitator as shown in Fig 2 .2(a) and 2.2(b) 
INTERNATIONAL JOURNAL OF INNOVATIVE RESEARCH IN ELECTRICAL, ELECTRONICS, INSTRUMENTATION AND CONTROL ENGINEERING Vol. 4, Issue 2, February 2016

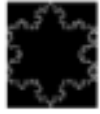

Fig. 2.2(a)
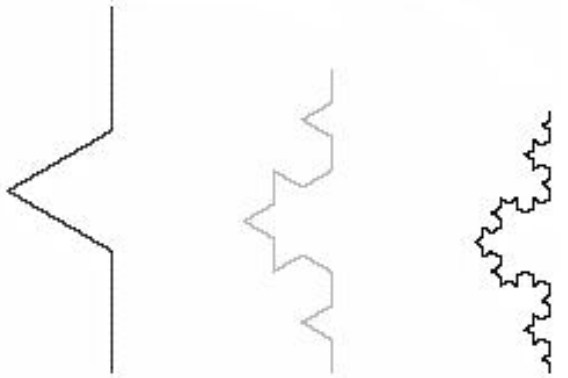

Fig. 2.2(b)

\subsection{Sierpinski Carpet fractal}

The construction of Sierpinski Carpet is similar to Sierpinski gasket with triangles replaced by squares as in 2.3(c). The starting geometry of the Carpet fractal antenna is the initiator square patch, and the successive iterations of fractal antenna is obtained by replacing each of the four straight sides of the starting structure with the generator.

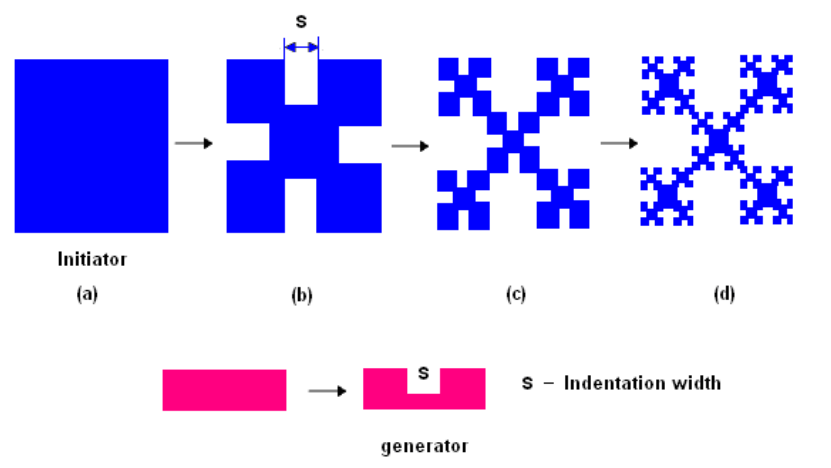

Fig. 2.3

\subsection{Hilbert Curve Fractal Antennas}

Hilbert curves can pack longer curves in a given area. This space filling property helps to fill electrically long antennas into small space. The multiple square curves as in fig .2.4 makes the operation of antenna at multiple frequencies, keeping the same electrical length and resonating at multiple frequencies.[12].A miniaturized antenna based on modified Hilbert curves suitable for implantable medical applications in a lossy environment was proposed in[13].
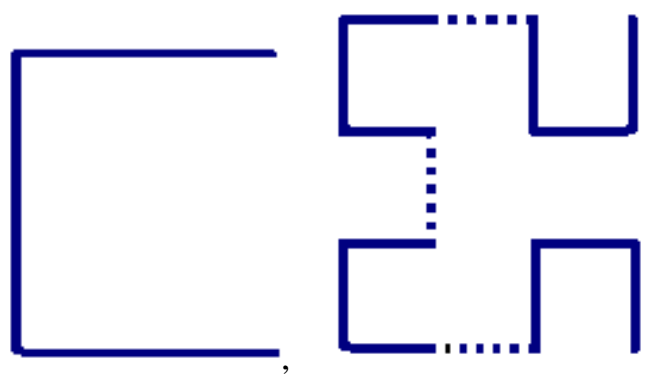

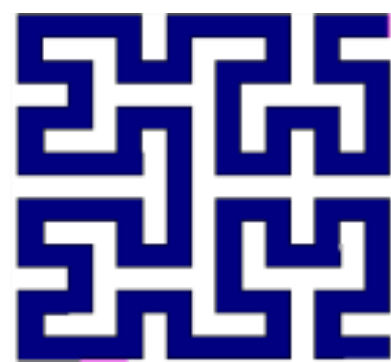

Fig. 2.4

\section{COMPARISON OF ANTENNAS BASED ON DIFFERENT FRACTAL GEOMETRIES}

A multiband fractal antenna based on the sierpinski carpet geometry was proposed in [4] with results shown in Table 1. Comparison of different antenna parameters based on different fractal geometries shows the results as in Table. 2

3.1Results of multiband fractal antenna based on the sierpinski carpet.

Table-1

\begin{tabular}{|l|l|l|}
\hline Resonant Frequency & Gain & Directivity \\
\hline $4.54 \mathrm{GHz}$ & $7.41 \mathrm{~dB}$ & 9.359 \\
\hline $4.706 \mathrm{GHz}$ & $1.251 \mathrm{~dB}$ & 4.491 \\
\hline
\end{tabular}

3.2 Comparison of different antenna parameters based on different fractal geometries.

Table-2

\begin{tabular}{|c|c|c|c|c|}
\hline $\begin{array}{l}\text { Sl. } \\
\text { No. }\end{array}$ & $\begin{array}{l}\text { Antenna } \\
\text { Structure }\end{array}$ & $\begin{array}{l}\text { Frequency } \\
\text { Band }\end{array}$ & Return Loss & Gain \\
\hline 1 & $\begin{array}{l}\text { Square } \\
\text { Fractale } \\
{[6,7]}\end{array}$ & $\begin{array}{l}3.0 \mathrm{GHz}, \\
07.49 \mathrm{GHz}, \\
09.68 \mathrm{GHz}, \\
11.21 \mathrm{GHz}\end{array}$ & $\begin{array}{l}22.20 \mathrm{db},- \\
18.70 \mathrm{~dB}\end{array}$ & $12.02 \mathrm{dBi}$ \\
\hline 2 & $\begin{array}{l}\text { Kotch } \\
\text { Fractal } \\
{[10,11,12]}\end{array}$ & $1-5 \mathrm{GHz}$ & $13-16 \mathrm{~dB}$ & $\begin{array}{l}2.98- \\
3.01 \mathrm{dbi}\end{array}$ \\
\hline 3 & $\begin{array}{l}\text { Circular } \\
\text { Fractal } \\
{[8,9]}\end{array}$ & $\begin{array}{l}3.0 \mathrm{GHz}, \\
4.275 \\
\mathrm{GHz}, \\
6.3 \mathrm{GHz}, \\
10.2 \mathrm{GHz}, \\
11.2 \mathrm{GHz} \\
\end{array}$ & $\begin{array}{l}15.5 \mathrm{db}- \\
20.2 \mathrm{db}, \\
20.4 \mathrm{db}- \\
20.8 \mathrm{db}, \\
25.5 \mathrm{db},-25 \mathrm{db}\end{array}$ & $>5 \mathrm{dBi}$ \\
\hline
\end{tabular}

\section{CONCLUSION}

A review on micro strip antenna using different fractal geometry is presented in this paper. The review shows that self-similar structure of fractals exhibits multiband property. And fractal antennas can be modified to bring about omnidirectional radiation pattern with good efficiency while maintaining other antenna parameter satisfactorily. Future studies are required to bring out a correlation between fractal geometries and antenna performance parameters.

\section{REFERENCES}

[1] Mandelbrot, B.B.: The Fractal Geometry of Nature, W.H. Freeman and Company, New York, 1983.

[2] Antenna Theory analysis and design-Constantine A. Balanis 
[3] H. O. Peitgen, H. Jurgens, and D. Saupe, Chao and Fractal: New Frontiers of Science, New York, Springer-verlag, Inc., 1992.

[4] Bandwidth and gain enhancement of multiband fractal antenna based on the sierpinski carpet geometry, ManasRanjan Jena , B.B. Mangaraj 2and Debasis Mishra Department of Electronics and Telecommunication Engineering, Veer SurendraSai University of Technology, India

[5] A Review of Various Fractal Geometries for Wireless Applications RupleenKaur 1, Satbir Singh 2 , Naveen Kumar 3 1,2 Dept. of ECE, Guru Nanak Dev University, Regional Campus Gurdaspur, Punjab, India Executive Director, Athal Services Private Limited, Chandigarh, India

[6] R.Yogamathi, S.Banu, A.Vishwapriya, "Design of Fractal Antenna for Multiband Applications", IEEE -31661, July 4-6, 2013.

[7] Mohammed, K. Ramasamy, T. Shanmuganantham, "A $2.45 \mathrm{GHz}$ Sierpinski Carpet edge-fed microstrip patch fractal antenna for WPT rectenna", IEEE International Multi-Conference on Systems Signal and Devices, pp 1-4, 2010.

[8] Raj Kumar, DhananjayMagar, K. Kailas Sawant, "On the design of inscribed triangle circular fractal antenna for UWB applications", International Journal of Electronics and Communication, pp 68-75, 2012.

[9] H.M. Alsabbagh, F.E. Mahmood, R.M. Edwards, J.A Brister, "AUWB fractal antenna for body area network applications", IEEEAntennas and Propagation Conference, pp 1-4,

[10] PiyushDalsania,Brijesh Shah, VedVyasDwivedi, "Analysis ofMultiband Behaviour on Square Patch Fractal Antenna", IEEEInternational Conference on Communication Systems and Network Technologies, pp 76-78, 2012

[11] S. Kohli, S.S. Dhillon, A. Marwaha, "Design and Optimization of Multiband Fractal Microstrip Patch Antenna for Wireless Applications", IEEE International Conference on Computational Intelligence and Communicati013.

[12] KamyaYekehYazdandoost and Ryuji Kohno, 2007, "An Antennafor Medical Implant Communications System", Proceedings of the37th European Microwave Conference,

[13] Study of Compact Hilbert Curve Fractal Antennas for Implantable Medical Applications S. Suganthi1, Dr. S. Raghavan 2, Dr. D. Kumar 3, Dr. J. Arputha Vijaya Selvi 